\title{
Surprisingly Weak Magnetism on Young Accreting Brown Dwarfs
}

\author{
A. Reiners* \\ Institut für Astrophysik, Georg-August-Universität, Friedrich-Hund-Platz 1, 37077 \\ Göttingen, Germany \\ Ansgar.Reiners@phys. uni-goettingen.de \\ and \\ G. Basri \\ Astronomy Department, University of California, Berkeley, CA 94720 \\ basri@berkeley. edu \\ and \\ U.R. Christensen \\ Max-Planck Institute for Solar System Research, Max-Planck-Strasse 2, 37191 \\ Katlenburg-Lindau, Germany \\ christensen@mps.mpg.de
}

\begin{abstract}
We have measured the surface magnetic flux on four accreting young brown dwarfs and one non-accreting young very low-mass star utilizing high resolution spectra of absorption lines of the FeH molecule. A magnetic field of 1-2 kG had been proposed for one of the brown dwarfs, 2MASS J1207334-393254, because of its similarities to higher mass $\mathrm{T}$ Tauri stars as manifested in accretion and the presence of a jet. We do not find clear evidence for a kilo-Gauss field in any of our young brown dwarfs but do find a $2 \mathrm{kG}$ field on the young VLM star. Our 3- $\sigma$ upper limit for the magnetic flux in 2MASS J1207334-393254 just reaches $1 \mathrm{kG}$. We estimate the magnetic field required for accretion in young brown dwarfs given the observed rotations, and find that fields of only a few hundred Gauss are sufficient for magnetospheric accretion. This predicted value
\end{abstract}

*Emmy Noether Fellow 
is less than our observed upper limit. We conclude that magnetic fields in young brown dwarfs are a factor of five or more lower than in young stars of about one solar mass, and in older stars with spectral types similar to our young brown dwarfs. It is interesting that, during the first few million years, the fields scale down with mass in line with what is needed for magnetospheric accretion, yet no such scaling is observed at later ages within the same effective temperature range. This scaling is opposite to the trend in rotation, with shorter rotation periods for very young accreting brown dwarfs compared with accreting solar-mass objects (and very low Rossby numbers in all cases). We speculate that in young objects a deeper intrinsic connection may exist between magnetospheric accretion and magnetic field strength, or that magnetic field generation in brown dwarfs may be less efficient than in stars. Neither of these currently have an easy physical explanation.

Subject headings: stars: low-mass, brown dwarfs - stars: magnetic fields

\section{Introduction}

Magnetic fields play an important role in astrophysics; in stars they regulate accretion of matter onto a star from the very beginning. Later they are the drivers of magnetic activity and regulate angular momentum and mass loss throughout the star's lifetime. Young ( T Tauri) stars can be affected by magnetic fields via two different mechanisms; (a) magnetospheric accretion, and (b) magnetic activity. Accreting T Tauri stars are usually referred to as "classical" (CTTS). The 10\% H $\alpha$ line width (White \& Basri 2003) is an indicator that can exclude the accretion scenario on stars with narrower $\mathrm{H} \alpha$ lines. However, in the presence of accretion only sparse information about magnetic activity can be drawn from emission lines.

Direct measurements of magnetic fields in $\mathrm{T}$ Tauri stars were carried out, e.g., by Johns-Krull et al. (1999), Yang et al. (2005), and Johns-Krull (2007). For low-mass magnetically active main-sequence stars magnetic fields are reported in, e.g., Johns-Krull \& Valenti (2000); Saar (2001) and Reiners \& Basri (2007). In both object classes, typical field strengths are on the order of a few kilo-Gauss. It is generally believed that the magnetic fields in active stars are generated through stellar dynamo processes. In sun-like stars, the dynamo is probably similar to the solar (interface) dynamo. In completely convective low-mass stars, however, a different type of dynamo is required to operate very efficiently because magnetic flux levels of several kilo-Gauss are apparently generated ubiquitously in active ultra-cool dwarfs (Reiners \& Basri 2007). T Tauri stars are completely convective as well, which im- 
plies that $\mathrm{T}$ Tauri stars could generate magnetic fields through the same dynamo mechanism operating in low-mass stars.

In the magnetospheric accretion model, accretion is funneled by a large-scale magnetic field. Gas flows from the disk to the central star forming an accretion shock and hot spots near the atmosphere of the accreting objects (e.g., Königl 1991; Shu et al. 1994; Hartmann et al. 1994). Outflows in CTTSs are also direcly related to magnetospheric infall (Hartigan et al. 1994; Königl \& Pudritz 2000). The strength of the magnetic field required for this mechanism was calculated by Königl (1991), Cameron \& Campbell (1993), and Shu et al. (1994). According to the scaling relations derived by these authors, the required surface magnetic field in an accreting object scales with mass and rotation period (with weaker magnetic field strength at more rapid rotation). The observation that surface rotation velocities in young brown dwarfs are comparable to those in more massive $\mathrm{T}$ Tauri stars (Mohanty et al. 2005) implies that rotation periods in accreting brown dwarfs are substantially shorter than in higher mass CTTS (because the brown dwarfs are smaller), therefore the magnetic field required to control the accretion in the brown dwarfs can be much weaker (see also Johns-Krull et al. 1999).

For this work, we selected a sample of slowly rotating, young late-M objects that are bright enough for high resolution spectroscopy. Mohanty et al. (2005) and Scholz \& Jayawardhana (2006) provide detailed information on a large sample of young very-low-mass objects. Our sample consists of five objects that are members of different star-forming regions with ages between 1 and 10 Myrs. Four of the targets are brown dwarfs. One target, 2MASSW J1207334393254 , is of particular interest because of the detection of accretion and an outflow.

\subsection{MASSW J1207334-393254}

2MASSW J1207334-393254 (hereafter 2MASS 1207-3932) is a $\sim 30 \mathrm{M}_{\text {Jup }}$ brown dwarf (Chauvin et al.|2005; Mohanty et al.|2007). It is a member of the $~ 8$ Myr old TW Hya association (Mohanty et al. 2003), and is surrounded by a disk (Mohanty et al. 2007; Riaz \& Gizis 2007). 2MASS 1207-3932 shows clear signs of accretion in bright asymmetric $\mathrm{H} \alpha$ emission (Mohanty et al. 2003), and rapid line emission variability probably due to rotation of the disk was found by Scholz \& Jayawardhana (2006). Whelan et al. (2007) discovered a bipolar outflow from 2MASS 1207-3932 in [O I] emission. Together, this provides strong arguments that 2MASS 1207-3932 is a low-mass analog to the more massive CTTS.

The accretion observed in 2MASS 1207-3932 clearly requires the presence of large-scale magnetic field. However, the strength of that field is not clear. A field much weaker than 
several kilo-Gauss could suffice to maintain the accretion process and mass outflow.

\section{Data}

Most of the data were taken at the W.M. Keck observatory with the HIRES spectrograph between 2006 and 2008. Our HIRES setup covers the wavelength range from below $\mathrm{H} \alpha$ $(6560 \AA)$ up to the molecular absorption band of $\mathrm{FeH}$ around $1 \mu \mathrm{m}$. We used a slit width of $1.15^{\prime \prime}$ achieving a resolving power of about $R=31000$. Data were cosmic-ray corrected, flatfielded, background subtracted, and wavelength calibrated using a ThAr spectrum. Data reduction was carried out using routines from the echelle package within the ESO/MIDAS distribution. Fringing is not an issue in spectra taken with the new HIRES CCD, even in very red spectral regions around $1 \mu \mathrm{m}$.

For 2MASS 1207-3932, the bulk of our data was obtained by us through a DDT run with UVES at the Very Large Telescope, ESO, Chile (PID 279.C-5026). Total exposure time was split into six exposures. We used a slitwidth of $1^{\prime \prime}$, which yields a resolving power of $R \approx 40000$. The detector was operated in $1 \times 2$ binning mode. We used the standard UVES setup centered at $860 \mathrm{~nm}$ in the red arm yielding spectral coverage from $6640 \AA$ to $10450 \AA$. Data were background-corrected and 2D-flatfielded in standard fashion using MIDAS-based reduction routines. Sky emission was removed by subtracting the mean of two sky spectra extracted above and below the target spectrum in direction perpendicular to dispersion.

Additional data from the ESO Archive1 was used for USco 55 (PID 067.C-0160, PI Guenther). This data was originally taken for radial velocity monitoring (see Guenther \& Wuchterl 2003) and was split into six exposures of 10 min duration each. It covers the same wavelength region as our UVES data for 2MASS 1207-3932. Our log of observations is given in Table1.

\section{Measuring rotation and magnetic flux}

The analysis of our spectra follows the strategy laid out in Reiners \& Basri (2006, 2007) and Reiners et al. (2009). To measure the projected rotation velocity $v \sin i$ and the magnetic flux $B f$ of our sample stars, we utilize the absorption band of molecular FeH close to $1 \mu \mathrm{m}$. We compare our data to spectra of the slowly rotating M-stars GJ 1002 (M5.5) and Gl 873 (M3.5). The magnetic flux of Gl 873 was measured to be $B f=3.9 k G$ (using an atomic FeI line; Johns-Krull \& Valenti 2000). In order to match the absorption strength of the

\footnotetext{
${ }^{1}$ http: //archive.eso.org
} 
target spectra, the intensity of the FeH absorption lines in the two comparison spectra is modified according to an optical-depth scaling (see Reiners \& Basri 2006). Note that in the magnetic flux measurement of our template star, Gl873, $B f$ is the weighted sum of several magnetic components used for the fit $(\Sigma B f)$. In our data, we cannot follow such an approach because (1) the spectral resolution of our data does not allow the differentiation of individual magnetic components, and (2) no information on the magnetic splitting pattern of FeH lines is used. Thus, with our method we can only scale the product $B f$ from the spectrum of Gl 873. We cannot provide results for $B$ and $f$ individually.

For the determination of the magnetic flux $B f$, we concentrate on relatively small wavelength regions that contain absorption lines particularly useful for this purpose, i.e. regions that contain some magnetically sensitive as well as magnetically insensitive lines. We determine the magnetic flux of our target stars by comparison of the spectral regions at 9946.0-9956.0 $\AA$ and 9972.0-9981.0 A. In Figs.1 3 we show the data and the quality of our fit in the top panels. The SNR of our data is between 20 and 30 in all targets.

For our analysis, we compare the spectra of young, cool stars and brown dwarfs to template spectra of older stars. We see no reason why differences in line formation, mainly differences in $\log$ g, would affect our measurement because all FeH lines belong to the same ro-vibrational transition. If FeH line formation is affected by a difference in log g, this would apply to all spectral lines. The strength of our differential technique is that we try to match magnetically sensitive and non-sensitive lines at the same time. Systematic effects would not result in fake magnetic field measurements but in an overall degradation of fit quality, which we do not see. An upper limit in our method implies that the scaled template of the inactive star did a good job of fitting the target spectrum. A preliminary study on the behaviour of $\mathrm{FeH}$ at different gravities was carried out by Wende et al. (2008) using 3Dhydrodynamical simulations. No systematic effect on the $\mathrm{FeH}$ lines, in particular between groups of magnetically more or less sensitive lines, was found.

\section{Results}

We show the fits to our data in the upper panels of Figs.1.3. We overplot the two extreme cases (a) without any magnetic field, and (b) strong magnetic flux with $B f \approx 4 \mathrm{kG}$. Template spectra are artificially broadened and scaled to match the absorption depth of the FeH lines in our sample targets (see Reiners \& Basri 2007). The lower panels of Figs, 13 show $\chi^{2}$-maps, i.e., the goodness of fit, $\chi^{2}$, as a function of projected rotational velocity, $v \sin i$, and total magnetic flux, $B f$. We mark the formal $3 \sigma$-region, $\chi^{2}=\chi_{\min }^{2}+9$, with a white line. In all cases, the values of the reduced $\chi^{2}, \chi_{\nu}^{2}$, is on the order of unity. As 
Table 1. Log of observations

\begin{tabular}{|c|c|c|c|}
\hline Name & UTC Date & $\begin{array}{c}\text { Exp.Time } \\
{[\mathrm{s}]}\end{array}$ & Instrument \\
\hline 2MASS $1207-3932$ & $\begin{array}{l}2006-05-06 \\
2007-09-30\end{array}$ & $\begin{array}{l}3600 \\
9800\end{array}$ & $\begin{array}{c}\text { Keck/HIRES } \\
\text { VLT/UVES }^{\mathrm{a}}\end{array}$ \\
\hline$\rho$ Oph-ISO 032 & 2008-05-18 & 4400 & Keck/HIRES \\
\hline USco DENIS 160603 & $\begin{array}{l}2006-05-12 \\
2008-05-17\end{array}$ & $\begin{array}{l}5400 \\
4800\end{array}$ & $\begin{array}{l}\text { Keck/HIRES } \\
\text { Keck/HIRES }\end{array}$ \\
\hline CFHT-BD-Tau 4 & 2008-09-20 & 4200 & Keck/HIRES \\
\hline USco 55 & $\begin{array}{l}2008-05-17 \\
2001-06 / 07\end{array}$ & $\begin{array}{l}3000 \\
3600\end{array}$ & $\begin{array}{l}\text { Keck/HIRES } \\
\text { VLT/UVES }^{\text {b }}\end{array}$ \\
\hline
\end{tabular}

${ }^{a}$ PID 279.C-5026

${ }^{b}$ PID 067.C-0160

Table 2. Results, some additional parameters and estimates on the magnetic flux using different assumptions

\begin{tabular}{lccccccccccc}
\hline \hline \multicolumn{1}{c}{ Name } & $\begin{array}{c}\text { Spectral } \\
\text { Type }\end{array}$ & $\begin{array}{c}v \sin i \\
{\left[\mathrm{~km} \mathrm{~s}^{-1}\right]}\end{array}$ & accretor $^{\mathrm{a}}$ & $\begin{array}{c}B f \\
{[\mathrm{kG}]}\end{array}$ & $\begin{array}{c}\mathrm{Age}^{\mathrm{b}} \\
{[\mathrm{Myr}]}\end{array}$ & $\begin{array}{c}T_{\text {eff }}^{\mathrm{c}} \\
{[\mathrm{K}]}\end{array}$ & $\begin{array}{c}M^{\mathrm{d}} \\
{[\mathrm{M} \odot]}\end{array}$ & $\begin{array}{c}R^{\mathrm{d}} \\
{\left[\mathrm{R}_{\odot}\right]}\end{array}$ & $\begin{array}{c}\log L^{\mathrm{d}} \\
{\left[\mathrm{L}_{\odot}\right]}\end{array}$ & $\begin{array}{c}B f_{\text {scale }} \\
\mathrm{kG}\end{array}$ & $\begin{array}{c}B_{\text {funnel }} \\
\mathrm{kG}\end{array}$ \\
\hline 2MASS 1207 - 3932 & $\mathrm{M} 8.0$ & $16_{-3}^{+1}$ & $\mathrm{Y}$ & $0.0^{+1.0}$ & 10 & 2700 & 0.037 & 0.26 & -2.48 & $2.0_{-1.2}^{+1.3}$ & 0.2 \\
$\rho$ Oph-ISO 032 & $\mathrm{M} 8.0$ & $12_{-3}^{+3}$ & $\mathrm{Y}$ & $0.6_{-0.6}^{+1.8}$ & 1 & 2700 & 0.032 & 0.44 & -2.10 & $1.4_{-0.8}^{+0.9}$ & 0.1 \\
UpSco DENIS 160603 & $\mathrm{M} 7.5$ & $14_{-1}^{+1}$ & $\mathrm{Y}$ & $0.0^{+0.4}$ & 5 & 2800 & 0.042 & 0.40 & -2.05 & $1.7_{-1.0}^{+1.1}$ & 0.1 \\
CFHT-BD-Tau 4 & $\mathrm{M} 7.0$ & $6_{-4}^{+2}$ & $\mathrm{Y} ?$ & $0.8_{-0.8}^{+1.0}$ & 2 & 2900 & 0.064 & 0.65 & -1.57 & $1.5_{-0.9}^{+1.0}$ & 0.2 \\
UpSco 55 & $\mathrm{M} 5.5$ & $9_{-1}^{+4}$ & $\mathrm{~N}$ & $2.3_{-1.4}^{+1.2}$ & 5 & 3050 & 0.095 & 0.52 & -1.68 & $1.9_{-1.1}^{+1.3}$ & 0.3 \\
\hline
\end{tabular}

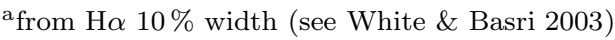

${ }^{\mathrm{b}}$ see Mohanty et al. (2005)

${ }^{\mathrm{c}}$ from spectral type according to Luhman et al. (2003)

d from age and $T_{\text {eff }}$ according to Baraffe et al. (1998) 
expected, the $3 \sigma$-regions are usually extended in $B f$ but relatively narrow in $v$ sin $i$. There is always a small tilt in the shape of these regions indicating the slight degeneracy between rotation and magnetic flux because both effects result in a broadening of spectral lines (but $B f$ only broadens a subset of all lines).

The results of our analysis for all stars are given in Table2, Values of $v \sin i$ and $B f$ are best fits from our $\chi^{2}$ analysis with formal $3 \sigma$-uncertainties. The uncertainties in $B f$ are usually on the order of $1 \mathrm{kG}$ reflecting the small effect of $B f$ on the spectral lines and the sometimes limited quality of our data. With this large an uncertainty we cannot rule out magnetic flux on the order of $1 \mathrm{kG}$ or lower. Nevertheless, we are able to distinguish between flux values on a kilo-Gauss scale. In Table2, we have also included estimates on stellar parameters including age, effective temperature, mass, radius, and bolometric luminosity (see below). Two estimates for magnetic flux from different perspectives are included as well, these will be explained in the following.

In only one of our five sample stars do we find magnetic flux on the kG-level; USco 55 shows magnetic flux of $B f \approx 2 \pm 1 \mathrm{kG}$. In all other objects, only upper limits on the order of $1 \mathrm{kG}$ are found. The preferred values for these would be several hundred Gauss; $1 \mathrm{kG}$ is the upper limit and it is unlikely that all objects would be at this three sigma maximum. In $\rho$ Oph-ISO 032, data quality is lower and the upper limit exceeds $2 \mathrm{kG}$.

\section{Discussion}

Although the uncertainties in magnetic flux measurements in these faint objects are quite large, the magnetic field detection in UpSco 55 stands in marked contrast to the nondetections in the other four targets. Magnetic flux on the $2 \mathrm{kG}$ level can be excluded for most of the four, and it is rather unlikely in all. In the following, we discuss implications and some possible explanations for this result.

The low-mass objects without magnetic flux detections represent the first objects of spectral type M6 and later in which magnetic flux is found to be weaker than a kilo-Gauss. Eight objects of spectral type M6 and later were investigated by Reiners \& Basri (2007), and all of them show strong magnetic flux. The main difference between our targets and the objects in Reiners \& Basri (2007) is their age, implying that they are of different mass. The four weakly magnetic objects of our sample can be considered brown dwarfs with quite some confidence, i.e., the mass estimates from age and spectral type are well below the theoretical threshold of hydrogen burning $\left(M=0.08 \mathrm{M}_{\odot}\right)$. UpSco 55 , on the other hand, is well above that boundary and hence is not a brown dwarf. In contrast to young brown 
dwarfs of spectral type late-M, a sample of late-M field stars at an average age of a few Gyrs (like in Reiners \& Basri 2007) have masses above the hydrogen burning limit as well. Hence, all other late-M objects with detected strong magnetic flux are not brown dwarfs; this is confirmed by the fact they all fail the lithium test (Basri 1998).

\subsection{Magnetospheric Accretion}

The second attribute that distinguishes these four brown dwarfs from older stars, and from UpSco 55, is the presence of magnetospheric accretion. A significant difference between young and old objects is that young objects are still surrounded by a disk to which they may be coupled through their magnetic field. The accretion of disk material in CTTS is believed to be governed by a magnetic field intercepting the disk and channeling the accretion flow onto the stellar surface. Königl (1991) provided the classic relation how the magnetic field is related to stellar mass and radius, accretion rate, and angular velocity (see his Eq.3). This relation is also connected to the inner radius of the disk, i.e. the radius where the disk is truncated. Using representative values for a model $\mathrm{T}$ Tauri star (see Königl 1991) with $M=0.8 \mathrm{M}_{\odot}, \dot{M}=10^{-7} \mathrm{M}_{\odot} \mathrm{yr}^{-1}, R=2.5 \mathrm{R}_{\odot}$, and $\Omega=1.7 \times 10^{-5} \mathrm{~s}^{-1}$, Königl (1991) predicts a magnetic field of kilo-Gauss strength on the surface of the star. The inner edge of the disk is then at a radius of $R_{\text {in }}=2 R_{*}$. Stellar magnetic fields of kilo-Gauss strength are indeed found in a number of $\mathrm{T}$ Tauri stars indicating that the model of Königl (1991) predicts field values of realistic magnitude. In fact, most TTS are found to have disks truncated further out than that, and even stronger surface fields (see e.g. Johns-Krull et al. 1999; Yang et al. 2005; Johns-Krull 2007). Cameron \& Campbell (1993) and Shu et al. (1994) derived similar relations for magnetic fields in accreting stars. While Cameron \& Campbell (1993) predict magnetic fields of comparable strength, the estimate of Shu et al. (1994) predicts magnetic fields about a factor of two weaker (cf. Johns-Krull et al. 1999).

Using the equation of Königl (1991), Scholz \& Javawardhana (2006) and Stelzer et al. (2007) predict the surface field strength in brown dwarfs to be about half as strong as in CTTS, which would be still in the kilo-Gauss regime. These authors make the assumption that the inner disk radius is constant at $R_{\text {in }}=2 \mathrm{R}_{*}$. This determines the angular rotation velocity of the objects. In fact, this assumption implies a surface rotation rate of $v=$ $40 \mathrm{~km} \mathrm{~s}^{-1}$ for their model star. Interestingly, from their calculation, Stelzer et al. (2007) predict a magnetic field of only $\sim 200 \mathrm{G}$ for 2MASS 1207-3932. However, for a more massive star they also derive a value of only $\sim 600 \mathrm{G}\left(M=0.8 \mathrm{M}_{\odot}, \dot{M}=10^{-8} \mathrm{M}_{\odot} \mathrm{yr}^{-1}, R=1.5 \mathrm{R}_{\odot}\right)$, which is substantially lower than the multikilo-Gauss fields found in CTTS. Stelzer et al. (2007) speculate that considerable uncertainties lead to this inconsistency. They suggest 
that both results, the $200 \mathrm{G}$ for the brown dwarf and the $600 \mathrm{G}$ for CTTS, underestimate the real fields by some factor. According to this argumentation, brown dwarfs should have fields a factor 2-3 weaker than CTTS, i.e. still on the order of a kilo-Gauss.

Instead of assuming a global underestimate of the field strengths, we can also estimate the magnetic field without the assumption of constant truncation radius, but assuming that the higher-mass star rotates at a lower surface velocity of only $v=10 \mathrm{~km} \mathrm{~s}^{-1}$ (as is typically observed). In this case, one finds a surface magnetic field of $B \sim 2.8 \mathrm{kG}$ for CTTS, which is more in line with observations. In other words, a somewhat slower rotation (hence larger truncation radius) easily pushes the surface magnetic field of a CTTS into the kilo-Gauss regime.

We can now apply the equations of Königl (1991) to our sample objects, without the assumption of a constant truncation radius. The lower limits of the measured surface rotation velocities (i.e., $i=90^{\circ}$ ) lead to upper limits of the magnetic fields of $B_{\text {funnel }} \sim 100-300 \mathrm{G}$. They are given in Table2, Note that these are upper limits $-B_{\text {funnel }}$ would be even lower if the stars are viewed under inclination angles $i<90^{\circ}$ implying more rapid rotation. This result is fully consistent with the non-detection of magnetic fields from our data. We conclude from this that our upper limits of magnetic flux in accreting brown dwarfs are not inconsistent with the magnetospheric accretion model. The ratio between surface magnetic flux necessary for magnetospheric accretion in CTTS of nearly one solar mass and in young accreting brown dwarfs is on the order of ten rather than two, given the observed values of surface rotation. There is an extensive literature of rotations for CTTS, and a good initial set of measurements for young brown dwarfs can be found in Mohanty et al. (2005).

While the weak magnetic fields are consistent with magnetospheric accretion, this does not explain the weakness of the fields, because magnetospheric accretion is thought to be a consequence of the presence of a magnetic field rather than a process that determines the magnetic field strength. Current models also assume that accretion applies no net torque on the object (Gosh \& Lamb 1979a, b). Hence, it is not clear how accretion could explain that young brown dwarfs have fields much weaker than their older stars of the same spectral type.

\subsection{Magnetic Flux Scaling with Energy Flux}

As mentioned above, the four late objects of our sample represent the first objects later than spectral type M5.5 on which no magnetic flux is detected. We can speculate why the magnetic field is rather weak on the surface of young brown dwarfs. A reasonable 
explanation might involve the fact that young brown dwarfs have not yet contracted, since the deuterium burning phase for these objects lasts about 10 Myr. Perhaps the magnetic field grows stronger as the star later shrinks. Christensen et al. (2009) presented a model in which the available energy flux determines the magnetic field strength of rapidly rotating objects (a few $\mathrm{km} \mathrm{s}^{-1}$ ). This model is successful in estimating magnetic fields in planets of our solar system, in field stars, and in T Tauri stars. One prediction of the model is that low-mass objects have weaker fields than high-mass objects, which is the very trend we observe in our small sample. We tested the hypothesis that energy flux of the objects explains the difference we see in magnetic flux (for more details, see Christensen et al. 2009). From the age and spectral type of the targets we estimate their mass, radius, and bolometric luminosity. We follow the conversion from spectral type to effective temperature by Luhman et al. (2003) and convert temperature and age into the other parameters according to the models of Baraffe et al. (1998). The predicted flux expressed in terms of mass, radius, and luminosity is

$$
B f_{\text {scale }} \sim\left(\frac{M}{M_{\odot}}\right)^{1 / 6}\left(\frac{L}{L_{\odot}}\right)^{1 / 3}\left(\frac{R}{R_{\odot}}\right)^{-7 / 6} \times 4.8_{-2.8}^{+3.2} \mathrm{kG},
$$

where the uncertainties are estimated along the lines discussed in Christensen et al. (2009, online supplementary information).

The magnetic flux predicted from an energy flux scaling, $B f_{\text {scale }}$, are given in Table2, They are in the range $1.4-2.0 \mathrm{kG}$ for all five targets including UpSco 55, for which a value of $1.9 \mathrm{kG}$ is predicted. The only object in which we find magnetic flux consistent with the expectation from an energy flux scaling is UpSco 55. According to this scaling, the differences in magnetic flux between UpSco 55 and the brown dwarfs should not exceed a few tens of percent.

\subsection{Constraints from Rotation and Activity}

There is also increasing evidence that even fully convective dynamos are sensitive to rotation period (Reiners \& Basri 2007). A potential way to explain the weak magnetic fields found in our young sample could be slow rotation. Observations have shown that in older brown dwarfs the rotation period decreases down to hours (Mohanty \& Basri 2003; Zapatero Osorio et al. 2006; Reiners \& Basri 2008). Thus, if magnetic flux generation is not saturated in a brown dwarf, it is likely to increase with age and might be rather low in young brown dwarfs. On the other hand, magnetic field generation obviously is effective in UpSco 55, which has a rather similar rotation period to the others. Its surface temperature 
and luminosity are rather similar to CFHT 4 (because that object is both younger and less massive). It is also a pre-main sequence object (unlike the stars in the older sample) and so has a similar interior structure and energy source compared to the brown dwarfs. Furthermore, the rotation periods of our targets are in the range of $1-6 \mathrm{~d}$ so that Rossby numbers are probably on the order of $\sim 10^{-2}$. This is well in the regime of saturated flux generation found in old M dwarfs (Reiners et al. 2009). Thus, slow rotation is a rather unlikely explanation for the lack of magnetic flux in young brown dwarfs.

Signs of magnetic activity are seen on both young accreting and non-accreting brown dwarfs, so the magnetic fields are strong enough to produce atmospheric heating in all cases. Grosso et al. (2007) find that young brown dwarfs achieve fractional X-ray luminosities that are about a factor of four smaller than for older main-sequence stars of similar effective temperature. These authors find no evidence for a difference in X-ray emission between accreting and non-accreting brown dwarfs, although CTTS tend to show less X-ray activity than non-accreting $\mathrm{T}$ Tauri stars (Güdel et al. 2006). From this perspective, the X-ray emission of young brown dwarfs could be expected to be about a factor of four below the level observed in older stars of the same spectral type. From the X-ray observations of other young brown dwarfs, one would expect a magnetic field of $0.5-1 \mathrm{kG}$ if X-ray emission is directly proportional to magnetic flux (an admittedly dubious proposition). Magnetic flux of a few hundred Gauss would still be consistent with our result.

\section{Conclusions}

Our main conclusion is that current theoretical predictions of magnetic fields required for funneled accretion are consistent with the first upper limit on the field strength in young brown dwarfs, i.e., with magnetic field strengths no larger than a few hundred Gauss. This implies that accretion in low mass objects requires much lower magnetic field strengths than in more massive stars, and that the actual field strength may not grow much beyond what is needed to regulate angular momentum during this part of brown dwarf evolution. Along with the regulation of angular momentum by stellar magnetic fields via disk-locking, this is a hint that the stellar fields may be regulated by disk accretion in return.

We find no magnetic fields in four accreting young brown dwarfs, but we detect a strong magnetic field in the non-accreting young low-mass star UpSco 55. The two differences between UpSco 55 and the other four targets are that (1) UpSco 55 has a mass above the hydrogen-burning limit, and (2) that it shows no accretion. Older stars (without accretion) above the hydrogen-burning limit but at similar temperature also show very strong fields. Thus, two ways emerge to explain the lack of magnetic flux in young brown dwarfs. First, 
magnetic fields may be somehow regulated by disk accretion, or, second, that the generation of magnetic flux is less effective in (young) brown dwarfs. In either case, the usual dependence on Rossby number seems to be violated, and apart from bulk rotation, it is hard to see how the presence of a disk could influence production of fields deep inside a star.

Johns-Krull (2007) finds that the situation may be complicated in part by how much of the total field is in a dipole component, but we appear to be seeing a general qualitative dependence between the presence of accretion and stellar magnetic flux. This should remain in place even after accretion has ended, until the object begins to contract and spin up. It will be important to make further direct field measurements on young brown dwarfs, both accreting and non-accreting, and also in old brown dwarfs.

It would have been easy (and not unexpected) for us to have found much stronger fields on our targets. To remain consistent with accretion regulation of angular momentum, however, we should then also have found a much lower rotation velocity. It is of interest, therefore, that to the extent we have been able to ascertain, in these fully convective objects there is a consistency of surface rotation velocities, and production of magnetic heating near the typical saturation limit, over a wide range of stellar ages, masses, and luminosities. This consistency is produced while balancing rather different accretion rates, truncation radii, rotation periods, and magnetic field strengths for different masses in young objects. It will be informative to follow these hints on the ways in which initial stellar parameters are tied to the conditions of star formation.

Based on observations collected at the European Southern Observatory, Paranal, Chile, PID 279.C-5026, taken from the ESO Science Archive Facility, PID 067.C-0160, and observed from the W.M. Keck Observatory, which is operated as a scientific partnership among the California Institute of Technology, the University of California and the National Aeronautics and Space Administration. We would like to acknowledge the great cultural significance of Mauna Kea for native Hawaiians and express our gratitude for permission to observe from atop this mountain. A.R. has received research funding from the DFG as an Emmy Noether fellow (RE 1664/4-1). G.B. thanks the NSF for grant support through AST00-98468.

\section{REFERENCES}

Baraffe, I., Chabrier, G., Allard, F., \& Hauschildt, P.H., 1998, A\&A, 337, 403

Basri, G., 1998, ASP Conf. Ser., 134, 394 
Burrows, A., Marley, M., Hubbard, W.B., Lunine, J.I., Guillot, T., Saumon, D., Freedman, R., Sudarsky, D., \& Sharp, C., 1997, ApJ, 491, 856

Cameron, A.C., \& Campbell, C.G., 1993, A\&A, 274, 309

Chauvin, G., Lagrange, A.-M., Dumas, C., Zuckerman, B., Mouillet, D., Song, I., Beuzit, J.-L., \& Lowrance, P., 2005, A\&A, 438, L25

Christensen, U.R., Holzwarth, V., \& Reiners, A., 2009, Nature, in press

Gosh, P., \& Lamb, F.K., 1979a, ApJ, 232, 259

Gosh, P., \& Lamb, F.K., 1979b, ApJ, 234, 296

Grosso,N., Briggs,K.R., Güdel,M., Guieu,S., Franciosini,E., Palla,F., Dougados,C., Monin,J.-L., Ménard,F., Bouvier,J., Audard, M., Tellesci, A., 2007, A\&A, 468, 391

Güdel, M., Padgett, D.L., \& Dougados, C., 2006, in Protostars and Planets V, ed., B. Reipurth, D. Jewitt, \& K. Keil, in press, arXiv:astro-ph/0609520

Guenther, E.W., \& Wuchterl., G., 2003, A\&A, 401, 677

Hartigan, P., Morse, J.A., \& Raymond, J., 1994, ApJ, 436, 669

Hartmann, L. Hewett, R., \& Calvet, N., 1994, ApJ, 426, 669

Johns-Krull, C.M., Valenti, J.A., \& Koresko, C., 1999, ApJ, 516, 900

Johns-Krull, C.M., \& Valenti, J.A.,, 2000, ASP Conf.Ser., 198, p.371

Johns-Krull, C.M., 2007, ApJ, 664, 975

Königl, A., 1991, ApJ, 370, L39

Königl, A., \& Pudritz, R.E., 2000, in Protostars and Planets IV, eds. V. Mannings, A.P. Boss, \& S.S. Russell (Tucson: Univ. Arizona Press), 759

Luhman, K., Stauffer, J., Muench, A., Rieke, G., Lada, E., Bouvier, J., \& Lada, C., 2003, ApJ, 593, 1093

Mohanty, S., Basri, G., 2003, ApJ, 583, 451

Mohanty, S., Jayawardhana, R., Barrado y Navascués, D., 2003, ApJ, 593, L109

Mohanty, S., Jayawardhana, R., \& Basri, G., 2005, ApJ, 626, 498 
Mohanty, S., Jayawardhana, R., Huélamo, N., \& Mamajek, E., 2007, ApJ, 657, 1064

Reiners, A., \& Basri, G., 2006, ApJ, 644, 497

Reiners, A., \& Basri, G., 2007, ApJ, 656, 1121

Reiners, A., \& Basri, G., 2007, ApJ, 684, 1390

Reiners, A., Basri, G., \& Browning, M., 2009, ApJ, in press, arXiv:0810.5139

Riaz, B., \& Gizis, J.E., 2007, ApJ, 661, 354

Saar, S.H., 2001, ASP Conf.Ser., 223, p.292

Scholz, A., \& Jayawardhana, R., 2006, ApJ, 638, 1056

Shu, F.H., Najita, J., Ostriker, E., Wilkin, F., Ruden, S., \& Lizano, S., 1994, ApJ, 429, 781

Stelzer, B., Scholz, A., \& Jayawardhana, R., 2007, ApJ, in press, arXiv:0707.1754

Wende, S., Reiners, A., \& Ludwig, H.-G., proceedings of the 15th Cambridge Workshop on Cool Stars, Stellar Systems, and the Sun, 2008, in press

Whelan, E.T., Ray, T.P., Randich, S., Bacciotti, F., Jayawardhana, R., Testi, L., Natta, A., \& Mohanty, S., 2007, ApJ, 659, L45

White, R.J., \& Basri, G., 2003, ApJ, 582, 1109

Yang, J.Y., Johns-Krull, C.M., \& Valenti, J.A., 2005, ApJ, 635, 466

Zapatero Osorio, M.R., Martín, E.L., Bouy, H., Tata, R., Deshpande, R., \& Wainscoat, R.J., 2006, ApJ, 647, 1405 

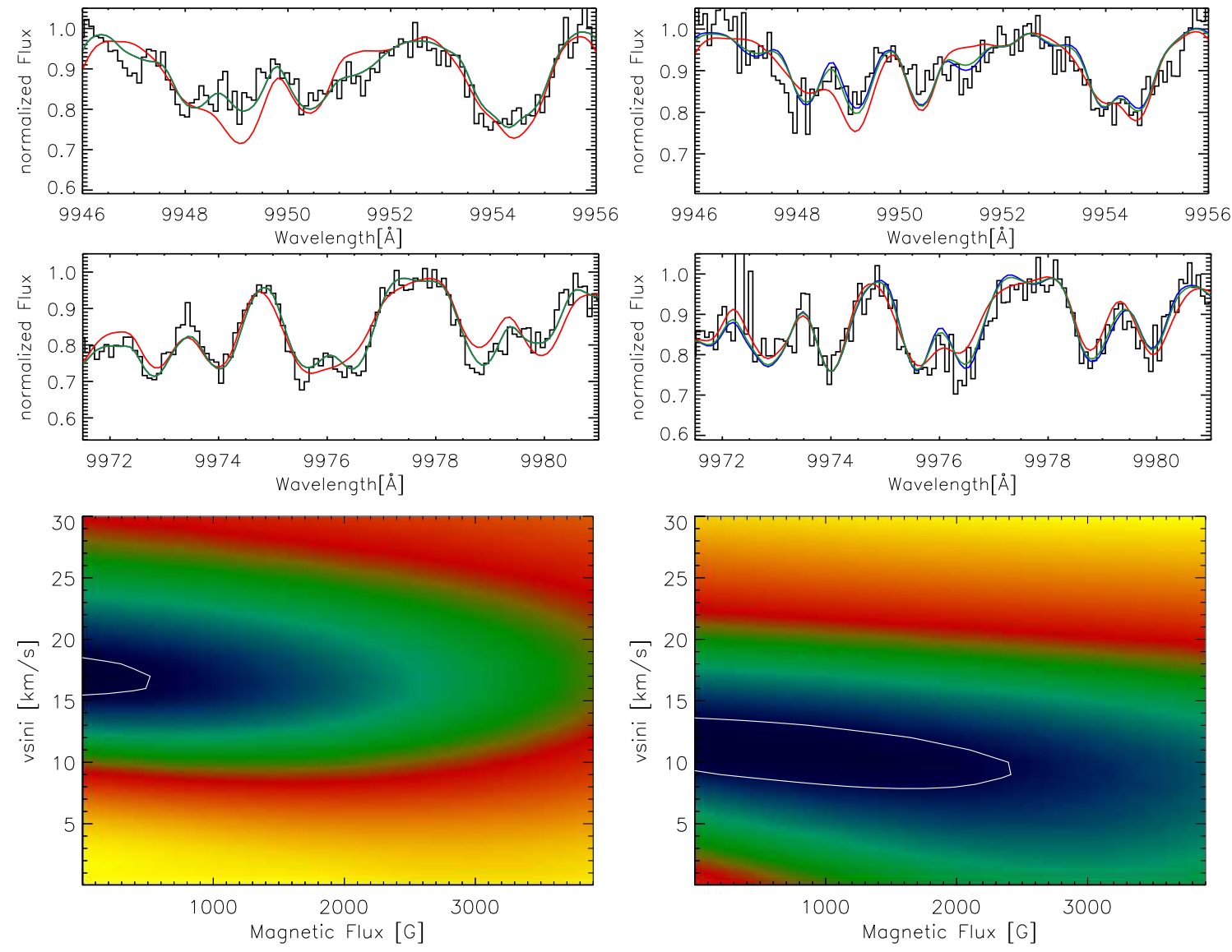

Fig. 1. - Data and fit (top panel) and $\chi^{2}$-landscapes (bottom panel) for 2MASS1207 (left) and ISO 032 (right). In the upper two panels, data are shown as black histograms. The three coloured lines show our fit for no magnetic field (blue line), strong magnetic flux $(B f \sim 4 \mathrm{kG}$, red line), and the best fit, which is an interpolation of the two (green line). In some cases, the green line is covering the blue line. In the bottom panel, dark and blue colour indicates low $\chi^{2}$ values, red and yellow colours show bad fit quality (high values of $\chi^{2}$. The white line shows the $3 \sigma$-level. 

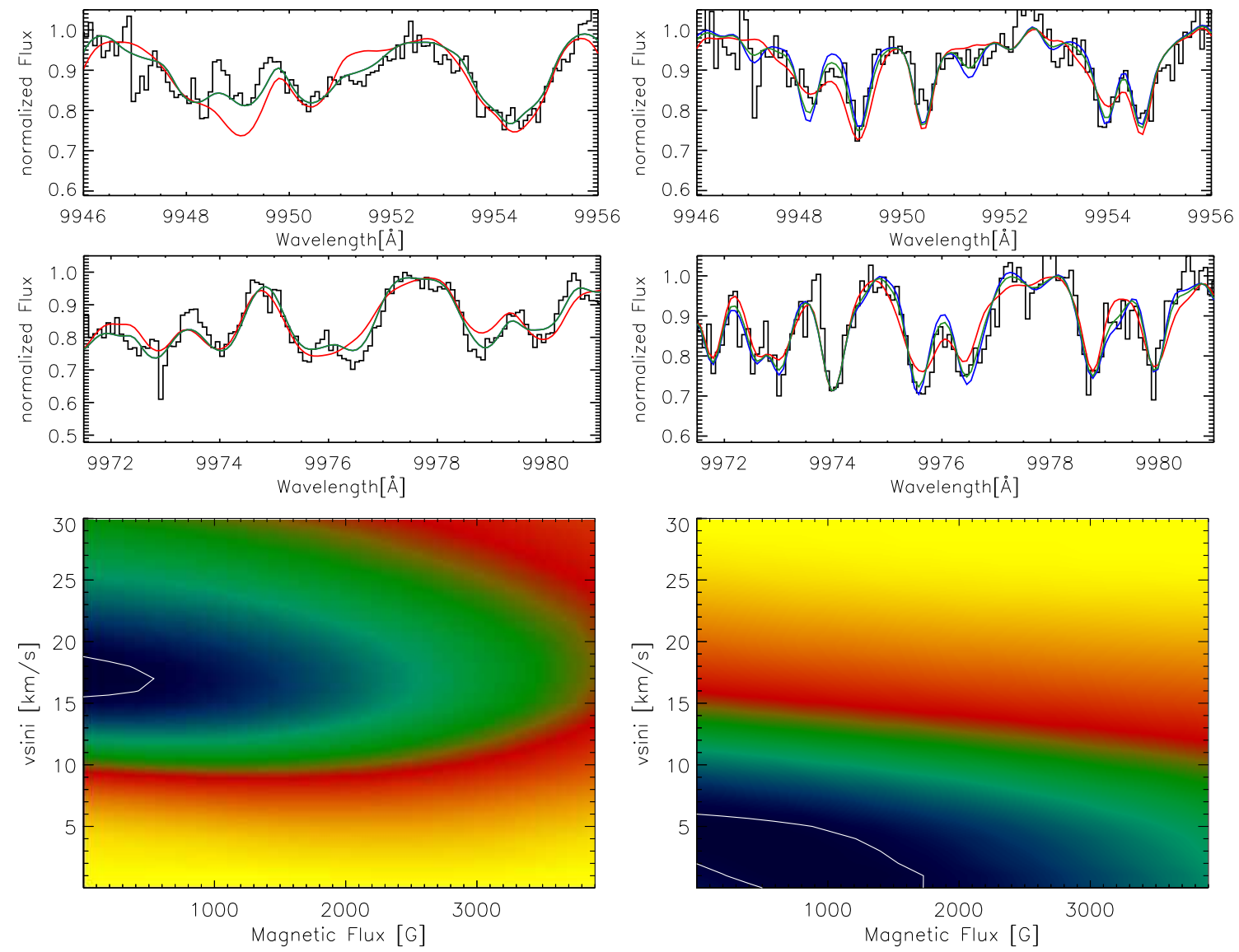

Fig. 2.- Data and fit (top panel) and $\chi^{2}$-landscapes (bottom panel) for UpSco DENIS 1606 (left) and CFHT 4 (right). See Fig.1 for more explanation. 

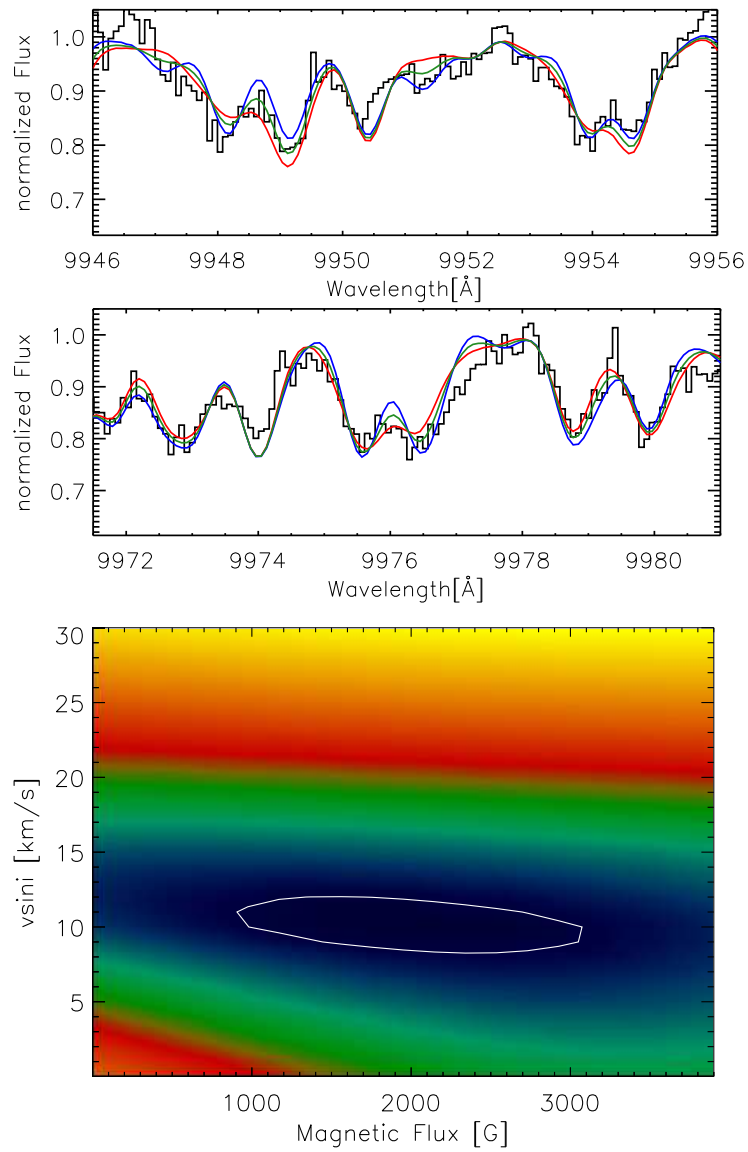

Fig. 3.- Data and fit (top panel) and $\chi^{2}$-landscape (bottom panel) for UpSco 55. See Fig.1 for more explanation. 